\title{
Blind Identification of Multichannels with Kalman Filter
}

\author{
Wanchun $\mathrm{Qu}^{1}$, Tiemin $\mathrm{Mei}^{1}$, Yulan $\mathrm{Hu}^{1}$ and Alfred Mertins ${ }^{2}$ \\ 1. School of Automatic and Electrical Engeering, Shenyang Ligong University, \\ Shenyang 110168 China;
}

2. Institute for Signal Processing, University of Luebeck, Luebeck 23562 Germany. meitiemin@163.com

\begin{abstract}
Keywords: multichannel,blind identification, cross relation, Kalman filter Abstract:A novel approach for blind multichannel identification is proposed on the basis of Kalman filter theory. Taking advantage of the cross relations between any pair of the multichannel outputs, the process and measurement equations are established in state space. The standard Kalman filter algorithm is simplified by exploiting the special zero observation vector which will leads to the homogenous iterative ways of the state vector and the filtered state-error correlation matrix. Simulations show that the convergence speed is significantly higher than those of LMS-like approaches.
\end{abstract}

\section{Introduction}

Channel identification is needed in many applications, including channel equalization [1,2], suppression of reverberation effects in audio processing [3,4]. Furthermore, the channel parameters can even be used to estimate the environmental scale [5,6]. In many applications, the channel identification has to be carried out blindly, this means that the channel parameters have to be estimated by exploiting no other information than the received signals. In this field, one of the pioneers is Godard, who proposed a family of Constant Modulus blind equalization algorithms which are widely used in blind channel equalization for cases of which the sources have constant envelope magnitudes [7].

Usually, the approaches for blind channel identification are classified into higher-order statistics (HOS) and second-order statistics (SOS) based algorithms [8]. HOS-based algorithms exploit HOS implicitly (for example, Godard's approach) or explicitly (for example, polyspectra-based algorithms). Some SOS-based approaches exploit the cyclostationarity of the channel input signal [9]. With the cyclostationarity of channel input signals and the oversampling of channel output signals, a single-input single-output (SISO) system is equivalent to a single-input multi-output (SIMO) system.

For non-minimum phase and finite impulse response (FIR) SISO systems, second-order statistics have been proven insufficient for blind channel identification without further assumptions on the input signal. However, the situation is different for SIMO models. For such systems, second-order statistics are sufficient for the blind identification of the channels. The channel impulse responses (CIRs) of a SIMO system can be identified by subspace decomposition [9] or by exploiting the cross relations (CR) between the SIMO output signals [1]. Based on the work in [1], both adaptive [10,11] and non-adaptive CR-based approaches [12] have been proposed. Adaptive 
algorithms are more attractive because of their inherent ability to track time-varying channels.

In this paper, a quick converging algorithmfor blind multichannel identification is proposed on the basis of Kalman filter theory.

\section{Problem Formulations}

For the derivation of the algorithm, a non-minimum phase and time-invariant FIR SIMO system is considered. Let $s(n)$ be the unknown input signal, and let $h_{i}(n)(i=1,2, \ldots, N)$ be the $N$ CIRs. The channel output signals, denoted by $x_{i}(n)(i=1,2, \ldots, N)$, are then given by:

$$
x_{i}(n)=\sum_{k=0}^{L-1} h_{i}(k) s(n-k)+v_{i}(n)
$$

where $v_{i}(n)$ is the observation noise, and $L$ denotes the length of the CIRs $h_{i}(n)$. The task of blind multichannel identification is to estimate $h_{i}(n)(i=1,2, \ldots, N)$ from the observed signals $x_{i}(n)(i=1,2, \ldots, N)$.

Whatever the multichannel impulse responses $h_{i}(n)(i=1,2, \ldots, N)$ are, the cross-relation (CR) between any pair of output signals holds as follows:

$$
x_{i}(n) * h_{j}(n)=x_{j}(n) * h_{i}(n)+\varepsilon_{i j}(n)(2)
$$

where * denotes the convolution operator; $\varepsilon_{i j}(n)=v_{i}(n) * h_{j}(n)-v_{j}(n) * h_{i}(n)$. For noise-free observations, the $\mathrm{CR}$ condition $x_{i}(n) * h_{j}(n)=x_{j}(n) * h_{i}(n)$ holds. But in practice, noise is always un-avoiding.

It is proved in[1] that if the channel impulse responses $h_{i}(n)(i=1,2, \ldots, N)$ are coprime in z-domain (i.e., if their z-transforms do not share common zeros), blind multichannel identification for non-minimum phase channel is possible on the basis of the cross-relations among the outputs of different channels.

\section{Algorithm Developments}

The Kalman-filter based algorithm will be established in this section. For this purpose,the multichannel identification problem is firstly expressed in state space with process equation and measurement equation, and then the algorithm is presented.

Let $\mathbf{h}_{i}=\left[h_{i}(0), h_{i}(1), \ldots, h_{i}(L-1)\right]^{\mathrm{T}}(i=1,2, \ldots, N)$. The required state vector is defined as $\mathbf{h}=\left[\mathbf{h}_{1}^{\mathrm{T}}, \mathbf{h}_{2}^{\mathrm{T}}, \ldots, \mathbf{h}_{N}^{\mathrm{T}}\right]^{\mathrm{T}}$. The process equation is expressed mathematically as

$$
\mathbf{h}(n+1)=\mathbf{F}(n+1, n) \mathbf{h}(n)+\mathbf{v}_{1}(n),(3)
$$

where $\mathbf{F}(n+1, n)$ is the state transition matrix of dimension $L N$-by- $L N$, the vector $\mathbf{v}_{1}(n)$ represents the process noise, which is usually modeled as a zero-mean, white-noise process.

For the construction of the measurement equation, the cross-relation equations in (2) are rewritten in matrix form as

$$
0=\mathbf{C}(n) \mathbf{h}+\mathbf{v}_{2}(n)
$$

where the $[N(N-1) / 2]-b y-L N$ matrix sequence $\mathbf{C}(n)$ is defined in Eq. (5); the $[N(N-1) /$ 2]-by-1vector $\mathbf{v}_{2}(n)$ is givenby: $\mathbf{v}_{2}(n)=\left[\varepsilon_{12}(n), \ldots, \varepsilon_{1 N}(n), \varepsilon_{23}(n), \ldots, \varepsilon_{2 N}(n), \ldots, \varepsilon_{(N-1), N}(n)\right]^{\mathrm{T}}$.

$$
\mathbf{C}(n)=\left[\begin{array}{ccccccc}
\mathbf{x}_{2}(n) & -\mathbf{x}_{1}(n) & 0 & \mathrm{~L} & \mathrm{~L} & \mathrm{~L} & 0 \\
\mathbf{x}_{3}(n) & 0 & -\mathbf{x}_{1}(n) & 0 & \mathrm{~L} & \mathrm{~L} & 0 \\
\mathrm{M} & \mathrm{O} & \mathrm{O} & \mathrm{O} & \mathrm{O} & \mathrm{O} & \mathrm{M} \\
\mathbf{x}_{N}(n) & 0 & \mathrm{~L} & \mathrm{~L} & \mathrm{~L} & 0 & -\mathbf{x}_{1}(n) \\
0 & \mathbf{x}_{3}(n) & -\mathbf{x}_{2}(n) & 0 & \mathrm{~L} & \mathrm{~L} & 0 \\
0 & \mathbf{x}_{4}(n) & 0 & -\mathbf{x}_{2}(n) & 0 & \mathrm{~L} & 0 \\
\mathrm{M} & \mathrm{O} & \mathrm{O} & \mathrm{O} & \mathrm{O} & \mathrm{O} & \mathrm{M} \\
0 & \mathbf{x}_{N}(n) & 0 & \mathrm{~L} & \mathrm{~L} & 0 & -\mathbf{x}_{2}(n) \\
\mathrm{M} & \mathrm{O} & \mathrm{O} & \mathrm{O} & \mathrm{O} & \mathrm{O} & \mathrm{M} \\
0 & \mathrm{~L} & \mathrm{~L} & \mathrm{~L} & 0 & \mathbf{x}_{N}(n) & -\mathbf{x}_{N-1}(n)
\end{array}\right] .
$$


where $\mathbf{x}_{i}(n)=\left[x_{i}(n), x_{i}(n-1), \ldots, x_{i}(n-L+1)\right]$.

According to the cross-relation equation (4), the measurement equation can be defined in state space as:

$$
\mathbf{y}(n)=\mathbf{C}(n) \mathbf{h}(n)+\mathbf{v}_{2}(n)
$$

where $\mathbf{C}(n)$ and $\mathbf{v}_{2}(n)$ are called the measurement matrix and measurement noise process, respectively; $\mathbf{h}(n)$ is the state vector at time $n$. The noise $\mathbf{v}_{2}(n)$ is approximatelymodeled as a zero-mean and temporally white noise process for high signal-to-noise ratio (SNR) observations. The vector $\mathbf{y}(n)$ in Eq. (6) is called the observation vector. How to define the observation vector $\mathbf{y}(n)$ is the key to solve our problem. According to the cross-relation equation (4) and the definition of measurement matrix (6), it is straightforward to find that

$$
\mathbf{y}(n)=\mathbf{0}(n=1,2, \ldots) .
$$

Such an observation vector will allow us to simplify the computation of the Kalman filter.

Obviously, the so-called observation vector $\mathbf{y}(n)$ cannot be directly 'observed' in practice, it is the direct result of CR relations. In contrary, the directly 'observed' signals are used to construct the measurement matrix.

For multichannel identification, it is natural to suppose that the process noise vector $\mathbf{v}_{1}(n)=\mathbf{0}$ and the state transition matrix $\mathbf{F}(n+1, n)=\mathbf{I}$, where $\mathbf{I}$ is an $L N$-by- $L N$ identity matrix, so the standard Kalman filter problem (Eqs. 3) and (6)) is simplified to the so-called unforced dynamical model [8]:

$$
\begin{array}{r}
\mathbf{h}(n+1)=\mathbf{h}(n), \\
\mathbf{y}(n)=\mathbf{C}(n) \mathbf{h}(n)+\mathbf{v}_{2}(n) .
\end{array}
$$

The standard Kalman filter algorithm (refer to [8]) is not only computationally inefficient but also quite memory demanding when the channels have long CIRs. However, for the unforced dynamical model in Eqs. (8) and (9), not only the computational burden, but also the memory demand can be reduced dramatically [8].

Furthermore, for the convenience of analyzing the computational efficiency, the basic formulae for the Kalman gain matrix $\mathbf{G}(n)$ andthe filtered state-error correlation matrix $\mathbf{K}(n)=\mathrm{E}\left[\boldsymbol{\alpha}(n) \boldsymbol{a}^{\mathrm{T}}(n)\right] \quad$ the filtered state-error: $\boldsymbol{\alpha}(n)=\mathbf{h}(n)-\hat{\mathbf{h}}\left(n \mid y_{n}\right)$, where $\left.y_{n}=\{\mathbf{y}(1), \mathbf{y}(2), \ldots, \mathbf{y}(n)\}\right)$ are presented as follows [8]:

$$
\begin{array}{r}
\mathbf{G}(n)=\mathbf{K}(n) \mathbf{C}^{\mathrm{T}}(n)\left[\mathbf{C}(n) \mathbf{K}(n) \mathbf{C}^{\mathrm{T}}(n)+\mathbf{Q}_{2}(n)\right]^{-1} \\
\mathbf{K}(n+1)=\mathbf{K}(n)-\mathbf{G}(n) \mathbf{C}(n) \mathbf{K}(n) .
\end{array}
$$

where $\mathbf{Q}_{2}(n)=\mathrm{E}\left[\mathbf{v}_{2}(n) \mathbf{v}_{2}^{\mathrm{T}}(k)\right]$. Because of the observation vector $\mathbf{y}(n)=\mathbf{0}$, the state vector is iteratively computed as

$$
\mathbf{h}(n+1)=\mathbf{h}(n)-\mathbf{G}(n) \mathbf{C}(n) \mathbf{h}(n) .
$$

A close examination on equations (11) and (12), it is found that they are iteratively computed in the same way despite the fact that $\mathbf{h}(n)$ is a vector and $\mathbf{K}(n)$ a matrix. This implies that each column of matrix $\mathbf{K}(n)$ complies with exactly the same computing rule of $\mathbf{h}(n)$, so if $\mathbf{h}(n)$ is initialized exactly as the first (or any) columnof $\left.\mathbf{K}(n)\right|_{n=0}$, it will converge in the same way of thecorresponding column of $\mathbf{K}(n)$. So the computation of equation (12) can be totally saved. The simplified Kalman filter algorithm is summarized in Table I.

TableI Simplified Kalman Filter Algorithm
(1) To form $\mathbf{C}(n)$ as defined in Eq. (5),
(3) $\mathbf{K}(n+1)=\mathbf{K}(n)-\mathbf{G}(n) \mathbf{C}(n) \mathbf{K}(n)$,
(2) $\mathbf{K}_{c}(n)=\mathbf{K}(n) \mathbf{C}^{\mathrm{T}}(n)$
(4) $\mathbf{h}(n)=$ the first column of $\mathbf{K}(n)$,
$\mathbf{G}(n)=\mathbf{K}_{c}(n)\left[\mathbf{C}(n) \mathbf{K}_{c}(n)+\mathbf{Q}_{2}(n)\right]^{-1}$,
$\mathbf{h}(n)=\mathbf{h}(n) / \max (|\mathbf{h}(n)|)$. 


\section{Simulation Results}

For the performance investigation, a speechsignal of 31744 samples with a sampling frequency of $f_{s}=8 \mathrm{kHz}$ was used as the input signal. For the SIMO system, two room impulse responses of 1000 taps were generated with the image method from [14]. The two simulated true CIRs are shown in Fig. 1. The longer effective length of these two CIRs is $L=942$ (the 58 zero taps before the first nonzero tap were discarded).

The normalized projection misalignment (NPM)[13]is used for evaluating the CIR's estimation accuracy:

$$
\operatorname{NPM}_{i}(n)=20 \log _{10}\left\{\left\|\mathbf{h}_{i}-\left[\mathbf{h}_{i}^{\mathrm{T}} \hat{\mathbf{h}}_{i}(n) / \hat{\mathbf{h}}_{i}^{\mathrm{T}}(n) \hat{\mathbf{h}}_{i}(n)\right] \hat{\mathbf{h}}_{i}(n)\right\| /\left\|\mathbf{h}_{i}\right\|\right\}
$$

where, $n$ is the iteration index, $\mathbf{h}_{i}$ and $\hat{\mathbf{h}}_{i}(n)(i=1,2, \ldots, N)$ are the trueand estimated CIRs, respectively. The NPM was computed during the iteration process.

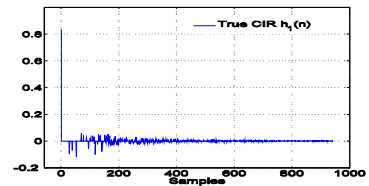

Fig . 1 The true CIRs generated with the image method. The longer effective length of these two CIRs is $L_{0}=942$.
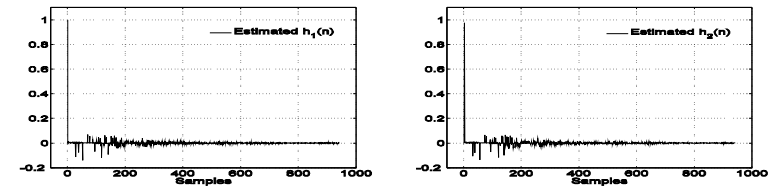

Fig. 2 The estimated CIRs for Case A: Noiseless signals, properly estimated order of CIRs: $L=L_{0}$.

Aiming at the performance investigation of the proposed algorithm, four different cases were studied as follows.

Case A:Noiseless signals and properly estimated orders of the CIRs (i.e., $L=L_{0}$ ) are considered. As the results shownin Fig. 2, the estimates are almost perfect. The dynamical NPMs are presented in Fig. 3 (Case A). As one can see, the algorithm converges after just 3000 iterations.

Case B: noiseless signals are used, but underestimated CIR orders are considered: $L=900<L_{0}$. The obtained NPM curve is shown in Fig. 3 (Case B). The estimation accuracy is degraded, but the convergence speed is not affected so much.

Case C:Noiseless signals and the overestimated CIR orders $\left(L=1100>L_{0}\right)$ are considered. The resulting NPM curve is shown in Fig. 4 (Case C). The performance is degraded, too, but it is a little better than in case B.

Case D: Noisy signals ( $\mathrm{SNR}=30 \mathrm{~dB}$ ) and properly estimated CIR orders $\left(L=L_{0}\right)$ are considered. The NPM curve is shown in Fig. 4 (Case D). As the figure shows, if the observed signals are contaminated by noise, the performance of the algorithm will be degraded. Especially, the convergence speed is slowed down.
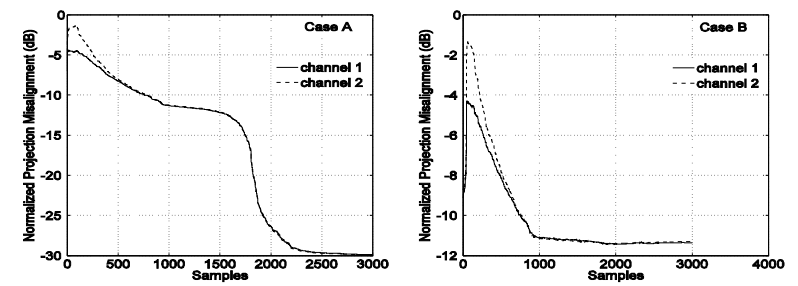

Fig. 3 The NPM curves for Cases A (left) and B(right).
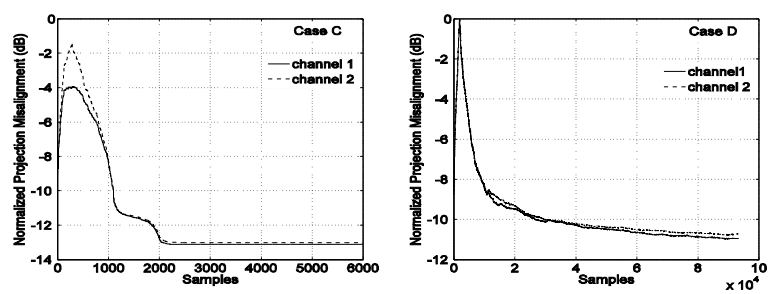

Fig. 4 The NPM curves for Cases C (left) and D(right).

\section{Conclusions}

Comparing with those LMS-like algorithms, the advantage of the proposed algorithm is its high convergence speed and high accuracy for clean multichannel output signals if the order of CIRs is 
correctly estimated. This is inherited from Kalman filtering theory because the Kalman filtering assumptions are almost satisfied for such a case.The performance of the algorithm will degrade for noisy signals and for cases in which the CIR orders are not correctly estimated. The reason is that the measurement noise process will go far from the Kalman filtering assumption if the observation noise is too strong or the channel order is not estimated correctly.

\section{Acknowledgements}

The project is funded by Liaoning Educational committee (LG201601); it is also supported by NSFC: 61373089.Corresponding author: Tiemin Mei, email: meitiemin@163.com

\section{References}

[1] G. Xu, H. Liu, L. Tong, T. Kailath, IEEE Trans. on Signal Processing, Vol.43(12) (1995),p. 2982-2992.

[2] L. Tong, S. Perreau, Proceedings ofIEEE, Vol.86(10) (1998),p. 1951-1968.

[3] T. Mei, A. Mertins, M. Kallinger,Proc.IEEE ICASSP,Taipei, Taiwan,(2009), p. 3745-3748.

[4] A. Mertins, T. Mei,M. Kallinger,IEEE Trans. Audio, Speech, and Language Processing, Vol. 18(2)(2010), p.249-259.

[5]N. Shabtai, B. Rafaely, Y. Zigel, 12th International Workshop on Acoustic Echo and Noise Control. Tel Aviv, Israel, (2010).

[6] J. Filos, E. Habets, P. A. Naylor,12th International Workshop on Acoustic Echo and Noise Control. Tel Aviv, Israel, (2010).

[7]D. N. Godard,IEEE Trans. on Communication, COM-28 (1980), p.1867-1875.

[8]S. Haykin, Adaptive filter theory (Third edition), Prentice-Hall, (1996), p. 302-321, p. 323-324.

[9] L. Tong, G. Xu, T. Kailath, IEEE Trans. on Information Theory, Vol. 40(2)(1994), p. 340-348.

[10] Y. Huang, J. Benesty, Proc. IEEEICASSP,Orlando, Florida, USA, Vol. II(2002), p.1637.

[11] Y. Huang, J. Benesty,IEEE Trans. on Signal Processing, Vol. 51(1) (2003), p. 11-24.

[12] E. Moulines, P. Duhamel, J. F. Cardoso, S. Mayrargue, IEEE Trans. on Signal Processing, Vol. 43(2)(1995), p. 516-526.

[13] M. K. Hasan, J. Benesty, P. A. Naylor, D. B. Ward,13th European Signal Processing. Antalya, Turkey,(2005).

[14] J. B. Allen,D. A. Berkley, J. Acoust. Soc. Amer., Vol. 65 (1979), p.943-950. 